\title{
Hydroxytyrosol (HT) Analogs Act as Potent Antifungals by Direct Disruption of the Fungal Cell Membrane
}

\author{
George Diallinas ${ }^{1 *}$, Nausica Rafailidou ${ }^{1}$, loanna Kalpaktsi², \\ Aikaterini Christina Komianou'2, Vivian Tsouvali', lliana Zantza ${ }^{2}$, Emmanuel Mikros², \\ Alexios Leandros Skaltsounis ${ }^{2}$ and loannis K. Kostakis ${ }^{2 *}$ \\ 1 Department of Biology, National and Kapodistrian University of Athens, Athens, Greece, ${ }^{2}$ Department of Pharmacy, \\ National and Kapodistrian University of Athens, Athens, Greece
}

OPEN ACCESS

Edited by:

Raffaella Balestrini,

Consiglio Nazionale delle Ricerche

(CNR), Italy

Reviewed by:

Giorgio Gnavi,

Università degli studi di Torino, Italy

Walter Chitarra,

Consiglio per la ricerca in agricoltura e l'analisi dell'economia agraria (CREA),

Italy

*Correspondence: George Diallinas diallina@biol.uoa.gr loannis K. Kostakis

ikkostakis@pharm.uoa.gr

Specialty section:

This article was submitted to Fungi and Their Interactions,

a section of the journal

Frontiers in Microbiology

Received: 25 June 2018 Accepted: 15 October 2018 Published: 06 November 2018

Citation:

Diallinas G, Rafailidou N, Kalpaktsi I, Komianou AC, Tsouvali V. Zantza I, Mikros E, Skaltsounis AL and Kostakis IK (2018) Hydroxytyrosol

(HT) Analogs Act as Potent Antifungals by Direct Disruption of the

Fungal Cell Membrane.

Front. Microbiol. 9:2624.

doi: 10.3389/fmich.2018.02624
Fungal infections constitute an emerging threat and a prevalent health problem due to increasing number of immunocompromised people and pharmacological or other treatments aiming at viral infections, cancer or allergies. Currently used antifungals suffer from inefficiency, toxic side effects and developing drug-resistance. Additionally, over the last two decades no new classes of antifungals have been approved, emphasizing the urgent need for developing a novel generation of antifungals. Here, we investigate the antifungal activity of a series of chemically synthesized Hydroxytyrosol $(\mathrm{HT})$ analogs. $\mathrm{HT}$ is one of the major phenolic compounds in olive oil, shown to possess radicalscavenging antioxidant, antiproliferative, proapoptotic and anti-inflammatory activities. No previous report has studied HT analogs as antifungals. We show that specific analogs have broad and strong antifungal activity, significantly stronger than the parent compound HT. Using Aspergillus nidulans as an in vivo cellular model system, we show that antifungal HT analogs have an unprecedented efficiency in fungal plasma membrane destruction. Importantly, antifungal HT analogs did not show toxicity in a mammalian cell line, whereas no resistance to HT analogs was obtained by standard mutagenesis. Our results open the way for the development of a novel, efficient and safer class of antifungals.

Keywords: fungal pathogens, Aspergillus nidulans, plasma membrane, antimicrobial, resistance

\section{INTRODUCTION}

In recent years, systemic fungal infections have emerged as an increasingly prevalent health problem (McCarthy et al., 2017). Infections are rising among immunocompromised patients, including individuals suffering from HIV/AIDS or diabetes mellitus, or following organ transplantations and immunosuppressive chemotherapy during cancer treatment (Low and Rotstein, 2011). The most clinically significant invasive opportunistic fungal pathogens belong to one of the four groups: Aspergillus, Candida, Cryptococcus and Pneumocystis, with the first two being the most important of all fungal pathogens (Köhler et al., 2017). Currently used antifungals include three major classes of drugs with different mechanisms of action: polyenes (disrupt fungal 
membranes), azoles (inhibit ergosterol biosynthesis), and echinocandins (inhibit synthesis of cell wall $\beta$-glucan) (Odds et al., 2003). However, all current antifungals suffer from inefficiency, toxic side effects, drug-drug interactions and developing drug-resistance (Arendrup, 2014; Cuenca-Estrella, 2014; Fairlamb et al., 2016). In addition, since 2001, no new classes of antifungals have been approved. This emphasizes the urgent and critical need for developing a novel generation of antifungals.

Pharmacological therapies for various bacterial or viral infections based on natural, mostly herbal, agents are among the most current therapeutic trends in medicine (Pan et al., 2013; Li and Weng, 2017). The antifungal activity of a large number of natural products is well documented (Di Santo, 2010). The most well-known structures exhibiting fungicide activity are polyenes, oligopeptides, terpenoids and macrolides, while different other natural products like flavonoids, alkaloids and phenolic acids have been also reported (Tasleem et al., 2009; Teodoro et al., 2015). Olive (Olea europaea), extracts has been reported for their antimicrobial and bacteriostatic activity since 1970 (Walter et al., 1973). Mild antifungal activity has been also described for olive leaf extracts (Korukluoglu et al., 2008) and the major olive phenolic compound oleuropein (Zorić et al., 2016). Recently, plant extracts and chemically synthesized related analogs from olive have also shown an antiprotozoan activity (Belmonte-Reche et al., 2016; Koolen et al., 2017). Hydroxytyrosol (HT), one of the major phenolic compounds in olive oil, has recently received particular attention because of its radical-scavenging, antiproliferative, proapoptotic and antiinflammatory activities, which seem to have a counteractive effect on carcinogenesis and other cellular malfunctions in animal trials and in vitro. Additionally, recent evidence has shown that HT and its analogs might be promising antibacterial, antiviral or antiprotozoan agents (Manna et al., 2005; Fernandez-Bolanos et al., 2008, 2012; Chillemi et al., 2010; Koolen et al., 2017; Thielmann et al., 2017; Robles-Almazan et al., 2018). However, no systematic effort has been invested in search of novel antifungals based on HT, except from some reports concerning yeast species (Pereira et al., 2007; Zoric et al., 2013), or HT analogs.

Based on some preliminary tests of our lab that showed a moderate antifungal effect of HT and some HT analogs on Aspergillus nidulans, here we systematically synthesize and test the antifungal activities of an extended series of novel HT analogs. The rationale of their synthesis was based mostly on the possible effect of the length and saturation of the fatty acid chain, and the substitution of the $\alpha$-carbon of the HT side chain. We show that several of the synthesized HT analogs have a very potent and broad antifungal activity against major fungal pathogens, such as A. fumigatus, A. flavus, Fusarium oxysporum, A. nidulans and Candida albicans. Importantly, we reveal that the antifungal activity of HT analogs is directly related to its rapid destructive effect on fungal plasma membranes, which in turn justifies why resistance mutants could not be isolated. Our work is expected to open the way for developing a new class of highly potent novel antifungals.

\section{RESULTS}

\section{Chemical Synthesis of HT Analogs}

Twenty three analogs of HT were synthesized as described in detail in the section "Materials and Methods" and in Supplementary Material (Supplementary Figure S1). The new compounds are lipophilic esters of HT bearing modifications on the $\alpha$-carbon of the catechol side chain. More precisely, the new compounds are categorized in 3 different series (Figure 1A). The compounds of the second series are simple esters of HT in the aliphatic hydroxyl group, whereas the compounds of the first series possess a carbonyl group on the $\alpha$-carbon of the catechol side chain. The compounds of the third series possess a hydroxyl group on the $\alpha$-carbon of the catechol side chain. In brief, chloride $\mathbf{1}$ was treated with the sodium salt of the appropriate acid to afford the required keto esters 212 (Figure 1B). The majority of the acids are commercially available, though the acids for the preparation of ester $\mathbf{9}$ was synthesized through a Wittig reaction of methyl 4-methylene cyclohexane carboxylate. Treatment of the previous compounds with triethylsilane in trifluoroacetic acid, provided the desired, fully reduced, lipophilic esters 13-20. It is noteworthy that this method was successful in yields up to $80 \%$, with high reproducibility and scale up to grams. Finally, partial reduction with hydrogenation over $\mathrm{Pd} / \mathrm{C}$, provided the hydroxyl derivatives 21-24, as racemic mixtures.

\section{HT Analogs Show High Potential as Antifungals}

The 23 chemically synthesized analogs of HT were tested for their antifungal potential against $A$. nidulans, A. fumigatus, A. flavus, C. albicans and Fusarium oxysporum, which are important fungal pathogens of animals and/or plants. The rationale for choosing A. nidulans was also based on its unique amenability as a model system for genetic and functional analysis, rather than its pathogenic profile, which would allow the investigation of the molecular mechanisms underlying of the antifungal action of HT analogs. All synthesized HT analogs were tested as described in Materials and methods. We tested all analogs on solid minimal media at physiological and optimal $\mathrm{pH}$ ranges (5.5-6.8) and temperatures $\left(25-37^{\circ} \mathrm{C}\right)$. The effect on C. albicans was also recorded in liquid fresh cultures at their logarithmic phase of growth. Importantly, similar results were obtained at the different $\mathrm{pH}$ tested and on complete media or minimal media. Notably, recorded apparent antifungal activities were significantly higher at $37^{\circ} \mathrm{C}$ compared to $25^{\circ} \mathrm{C}$ (see also later).

Figure 2 and Table 1 highlight our findings and reflects the outcome obtained through at least three independent growth tests that showed practically identical results. Nine HT analogs $(2,4,5,10,11,15,16,18$ and 19), shown in Figure 3 had strong antifungal activity against $A$. nidulans at $37^{\circ} \mathrm{C}$, mostly evident at $400 \mu \mathrm{M}$, and six of them $(2,5,11,15,16$ and 19) were also very active at $25^{\circ} \mathrm{C}$. Most of the same nine analogs also had strong antifungal activity against other fungi tested, at $25^{\circ} \mathrm{C}$ and $37^{\circ} \mathrm{C}$ (Figure 2A, Table $\mathbf{1}$ and test not shown). In particular, 
A<smiles>OCCc1ccc(O)c(O)c1</smiles><smiles>[R]C(=O)OCCc1ccc(O)c(O[AsH2])c1</smiles><smiles>CC(C)C</smiles>

1<smiles>CCC(=O)c1ccc(O)c(O)c1</smiles><smiles>[2H]C(=O)O</smiles><smiles>[CH]1CCC1</smiles><smiles>[O-]C1CCCCO1</smiles><smiles>[Z12]CCC(O)c1ccc(O)c(O)c1</smiles>

FIGURE 1 | (A) Structures of Hydroxytyrosol (HT) and general formula of the compounds (B) Reactions and Conditions: a) sodium salt of suitable acid, DMF, $70^{\circ} \mathrm{C}$; b) $\mathrm{Et}_{3} \mathrm{SiH}, \mathrm{CF}_{3} \mathrm{COOH}$, r.t.; c) $\mathrm{H}_{2}, 10 \% \mathrm{Pd} / \mathrm{C}$, t-BuOH, 50 psi, r.t.

all nine analogs were extremely toxic to A. fumigatus, leading to total or extremely severe inhibition of growth at $100 \mu \mathrm{M}$. A. flavus proved to be the most resistant fungus among those tested, but still several analogs were highly inhibitory for its growth $(2,5,11,15,16$ and 19). Best antifungal agents against F. oxysporum proved to be analogs $2,5,11$ and 15. All analogs, at $100 \mu \mathrm{M}$, severely inhibited C. albicans growth in liquid cultures (Figure 2B) or on solid minimal media (not shown) at $37^{\circ} \mathrm{C}$. When liquid cultures were left to grow for more than $24 \mathrm{~h}$ after the initial addition of HT analogs, growth $C$. albicans resumed in several cases, but not in the presence of analogs 4 or 15 (Supplementary Figure S2). This indicates that these analogs had the strongest cytotoxic effect or that these compounds were the most stable under the relative experimental conditions. Overall, several synthesized analogs of HT have a dramatically increased antifungal activity, compared to the "mother" natural compound (HT), and importantly, the most of active of them are comparable to Amphotericin B (Supplementary Figure S3). The rationale of this latter comparison becomes apparent later.

\section{Antifungal HT Analogs Show Variable Antibacterial Activities}

The HT analogs with the highest antifungal activity were also tested, at a range of 100-200 $\mu \mathrm{M}$, in fresh exponentially growing bacterial cultures in order to gain further insight on their mode of action and as a first step for investigating their potential use as broad range antimicrobials. Figure 4 shows the results obtained with E. coli and B. subtilis, 
A
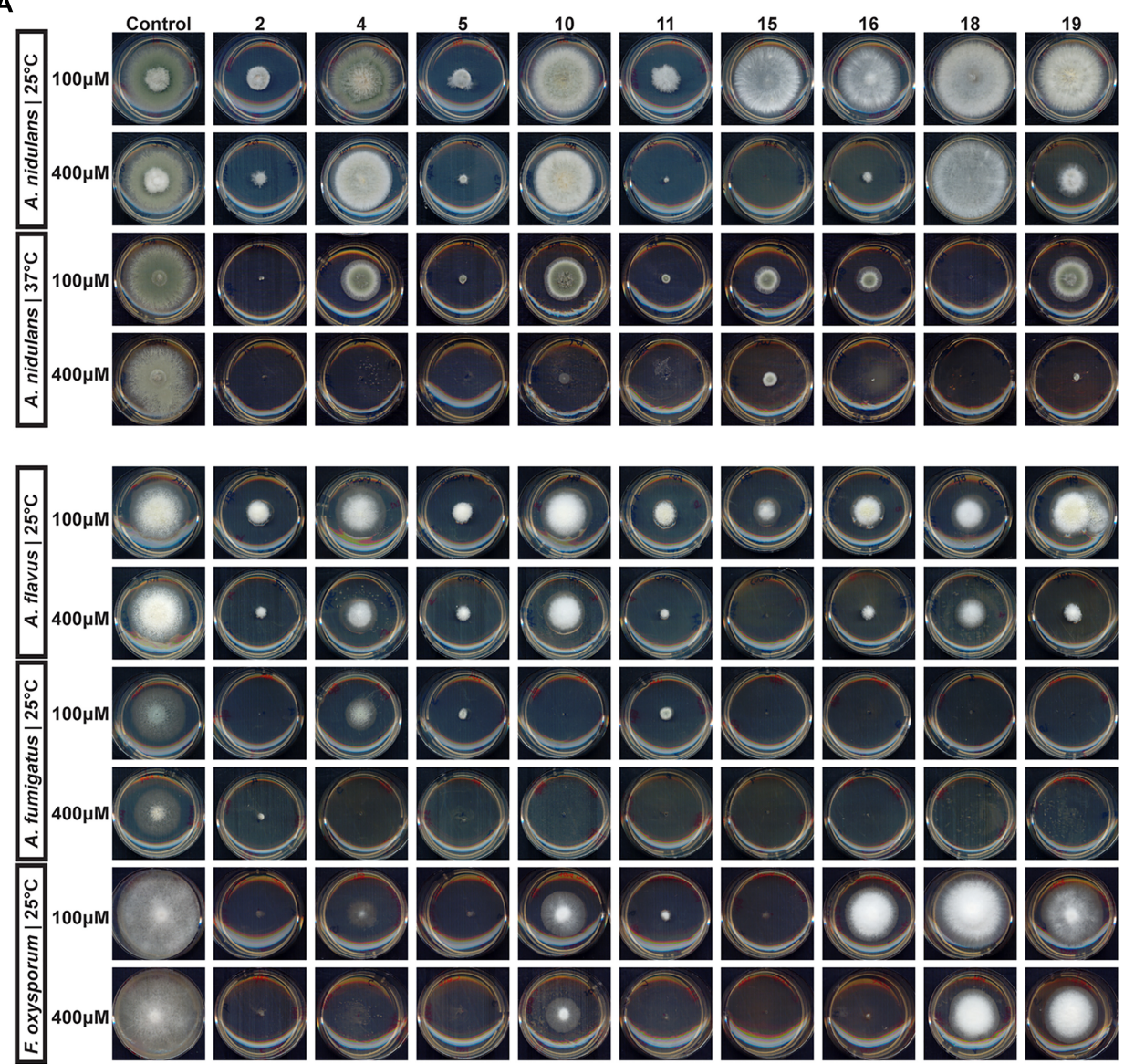

B

Growth curve of Candida albicans with $100 \mu \mathrm{M}$ of added HT analogs
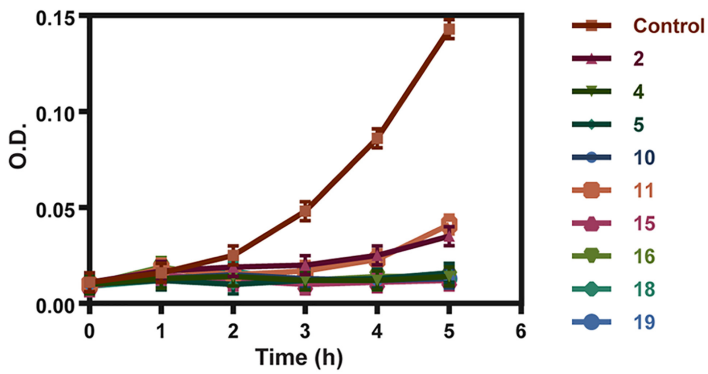

FIGURE 2 | In vivo evaluation of HT analogs as antifungals (A) Growth tests showing the antifungal activity of certain HT analogs against pathogens A. flavus A. fumigatus, F. oxysporum and A. nidulans. Growth on two concentrations of HT analogs for each microorganism is shown. (B) Growth curve of C. albicans. O.D stands of Optical Density at $600 \mathrm{~nm}$ of liquid cultures recorded hourly. Analogs were added to the cultures at $200 \mu \mathrm{M}$. Control stands for cultures were only DMSO solvent was added in the cultures, at the same concentrations as the analogs.

as typical representatives of $\mathrm{G}-$ and $\mathrm{G}+$ bacteria. All analogs were highly toxic to $B$. subtilis at $100 \mu \mathrm{M}$, but not at all to E. coli. Pseudomonas species showed differential growth behavior, with $P$. aeruginosa being fully resistant, but $P$. fluorescens highly sensitive at $200 \mu \mathrm{M}$ (Supplementary Figure S4). Additional bacterial species were also tested (Klebsiella, Enterococcus Staphylococcus, Streptococcus and other $G$ - enterobacteria) showing varying degrees of sensitivity to HT analogs, but this will be reported elsewhere as the present work is directed toward the discovery of novel antifungals.

\section{Antifungal HT Analogs Disrupt the Structure and Function of $A$. nidulans Plasma Membrane}

The non-dependence of the antifungal action of the HT analogs on whether minimal or complete media are used, or the $\mathrm{pH}$ 
<smiles>O=C(COC(=O)C12CC3CC(CC(C3)C1)C2)c1ccc(O)c(O)c1</smiles><smiles>CCCCCCC(=O)OCC(=O)c1ccc(O)c(O)c1</smiles><smiles>O=C(CC12CC3CC(CC(C3)C1)C2)OCC(=O)c1ccc(O)c(O)c1</smiles><smiles>CC(C)C1CCC(C(=O)OCC(=O)c2ccc(O)c(O)c2)CC1</smiles>

10<smiles>O=C(CO)c1ccc(O)c(O)c1</smiles><smiles>CCCCCCCC(=O)OCCc1ccc(O)c(O)c1</smiles>

11

15<smiles>CC(CC(=O)OCCc1ccc(O)c(O)c1)CC(C)C1CCC(C(=O)OCCc2ccc(O)c(O)c2)CC1</smiles>

FIGURE 3 | Specific HT analogs showing strongest antifungal activity.

TABLE 1 | Approximate concentrations of $\mathrm{HT}$ analogs that lead to $50 \%$ reduction of fungal growth $\left({ } C_{50}\right)$.

\begin{tabular}{|c|c|c|c|c|c|c|c|c|c|c|}
\hline & HT & 2 & 4 & 5 & 10 & 11 & 15 & 16 & 18 & 19 \\
\hline A. nidulans $25^{\circ} \mathrm{C}$ & $>400$ & $<100$ & $<200$ & $<100$ & $<200$ & $\sim 100$ & $<200$ & $<200$ & $<200$ & $<200$ \\
\hline A. flavus & $>400$ & $<100$ & $>100$ & $<100$ & $>100$ & $<100$ & $<100$ & $\sim 100$ & $\sim 100$ & $>100$ \\
\hline F. oxysporum & $>400$ & $<50$ & $<50$ & $<50$ & $\sim 100$ & $<50$ & $<50$ & $<200$ & $<200$ & $<200$ \\
\hline C. albicans & $>400$ & $\sim 100$ & $\sim 100$ & $\sim 100$ & $\sim 100$ & $\sim 100$ & $\sim 100$ & $\sim 100$ & $\sim 100$ & $\sim 100$ \\
\hline
\end{tabular}

$1 C_{50}$ values shown correspond to the approximate $\mu \mathrm{M}$ concentration of the compounds that reduce the diameter of colony growth by $50 \%$ after $4-6$ days at $37^{\circ} \mathrm{C}$, on standard agar minimal media. IC 50 are estimated by testing growth in the presence of a range of $\mu \mathrm{M}$ concentration (i.e., 0, 50, 100, 200, 400, 500, and 1000 $\mu$ M). The values shown were estimated by at least three independent experiments, which showed no significant variation (SD $=20 \pm 5 \%$ ). For one of the most active analogs, 15 , additional experiments performed at a lower concentration range $(5,10,20,50$, and $100 \mu \mathrm{M})$, led to a more precise estimation of $I C_{50}$, which corresponds to $17 \mu \mathrm{M}$. Most analogs led to total inhibition of growth at 100-400 $\mu \mathrm{M}$ against all fungi tested (see Figure 2).

range, when this was kept within limits proper for fungal growth, suggested that these compounds might be either taken up by non-facilitated diffusion, or exert their activity directly without the need to enter the cell (i.e., on the cell wall or the plasma membrane). The relatively increased activity observed at $37^{\circ} \mathrm{C}$ vs. $25^{\circ} \mathrm{C}$ in the case of A. nidulans (see Figure 2) does not distinguish between the two possibilities, as increased membrane fluidity at a higher temperature would favor diffusion, as well as, binding of hydrophobic HT analogs in specific lipids of the fungal membrane. Thus, to investigate this issue directly we followed the effect of all antifungal HT analogs on the microscopic morphology and the plasma membrane (PM) of A. nidulans hyphae, using Brightfield and Epifluorescence microscopy, respectively. For investigating the effect on the plasma membrane we used strains expressing two GFP-tagged plasma membrane transporters, namely the UapA uric acid-xanthine transporter (Pantazopoulou et al., 2007) or the FurA allantoin transporter (Krypotou et al., 2015). Figure 5 summarizes our results. Most HT analogs, when added at final concentration as low as $37.5 \mu \mathrm{M}$, for $0-30 \mathrm{~min}$, had a rapid and prominent effect on plasma membrane integrity, reflected in dramatic reduction of transporter-associated peripheral GFP fluorescence signal and concomitant appearance of static, noncortical cytosolic membrane fluorescent aggregates (most evident with analogs $2,5,10,11,16$ or 18). The great majority of these cytosolic fluorescent aggregates did correspond to vacuoles or other known endomembrane compartments, as evidenced by CMAC or FM4-64 staining (not shown). Under brightfield light we did not notice any dramatic modification that would be compatible with disruption of the cell wall or overall hyphae morphology (see also Supplementary Figure S5), despite a visible increase in vacuole number and size. These 


\section{E.coli with $200 \mu \mathrm{M}$ of added $\mathrm{HT}$ analogs}
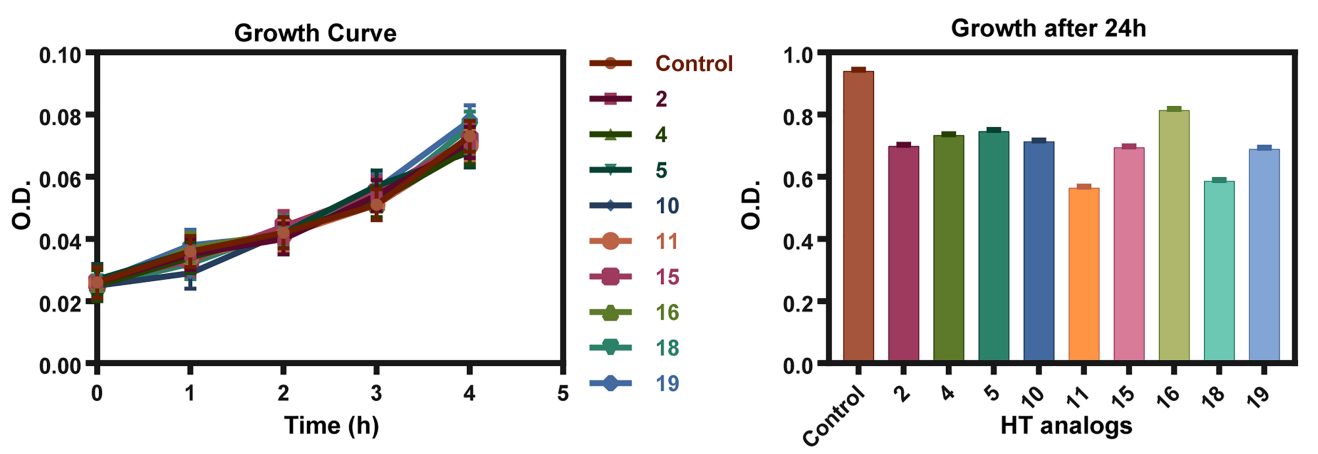

B. subtilis with $100 \mu \mathrm{M}$ of added HT analogs
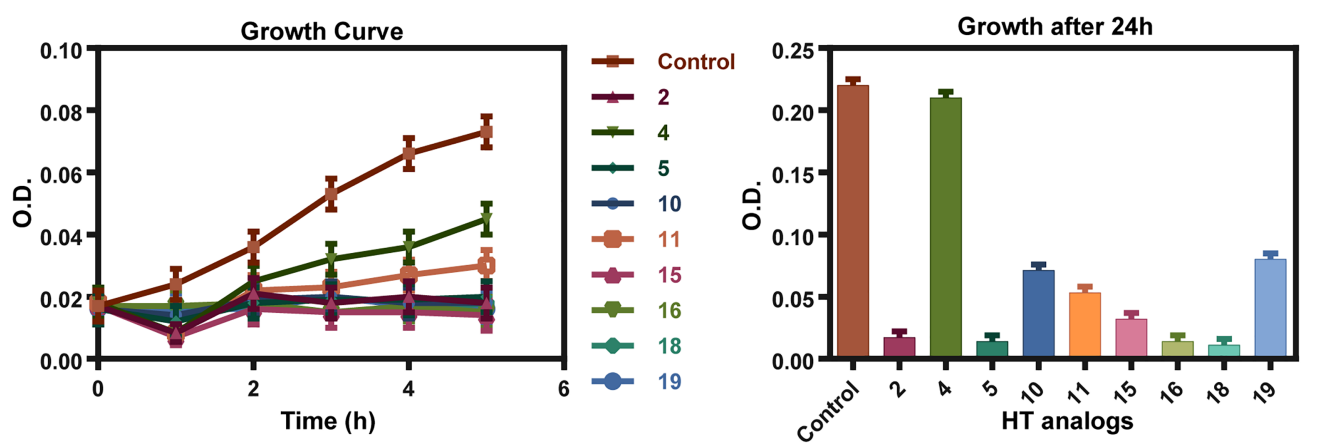

FIGURE 4 | Antibacterial action of HT analogs against E. coli and B. subtilis. Growth curves on the left show O.D. values recorded hourly at $600 \mathrm{~nm}$. Column bar graphs on the right show growth after $24 \mathrm{~h}$ after the $\mathrm{HT}$ analogs addition, at $600 \mathrm{~nm}$. Control stands for samples where only the solvent DMSO was added at a concentration identical to the one used for the dissolved analogs.

effects were practically immediate, becoming evident in 1 5 min, which also somehow excludes the idea that HT analogs act primarily by metabolic inhibition of an enzyme. In general, the relative strength of the detrimental effect of the different HT analogs on the PM was variable, with some analogs leading to total apparent disintegration, while others led to significant but not total, of the PM in $10 \mathrm{~min}$. The concentration used to test the analogs was $37.5 \mu \mathrm{M}$ in order to avoid any effect of DMSO (the solvent used) on the stable localization of transporters in the PM, as we have noticed that DMSO concentrations $>50 \mu \mathrm{M}$ elicit a degree of endocytic turnover for most transporters studied in our lab (unpublished observations).

To further examine the nature of the effect of HT analogs on the PM, we also performed direct transport assays of radiolabeled metabolites, imported by specific transporters, in the presence of excess analogs. Transport assays used measure initial uptake rates (at $60 \mathrm{~s}$ ) of radiolabeled substrates in germinated conidiospores (Krypotou and Diallinas, 2014). In particular, we tested the uptake of radiolabeled xanthine, which is specifically transported by two uptake systems, the UapA $(\sim 70 \%)$ and UapC $(\sim 30 \%)$ transporters (Pantazopoulou and Diallinas, 2007; Krypotou and Diallinas, 2014). Results are summarized in Figure 6. HT analogs $2,4,5,10,11,15$ and 16 reduced xanthine uptake to $\sim 20-$ $40 \%$ of the control sample (only DMSO added), whereas the rest (18 and 19) showed less inhibitory effect. Overall, these results strongly suggested that most HT analogs tested had a rapid negative effect on fungal transport systems. Given that the relevant xanthine transporters, UapA and UapC, as most fungal transporters, are $\mathrm{H}^{+}$symporters, our results can in principle be explained by two scenarios; either the analogs lead to rapid disorganization of the PM, or they led to a rapid depolarization of the membrane, acting as direct $\mathrm{H}^{+}$gradient uncouplers. However, the latter case seems unlikely, or secondary, under the light of the microscopic analysis shown in Figure 5, which directly confirmed the dramatic effect of all HT analogs tested on the PM within some minutes after their addition to the fungal cultures. Additionally, the strength of inhibition of transportermediated xanthine uptake by the different HT analogs tested was in good agreement with the results obtained following their effect on PM integrity (Figure 5) and their in vivo antifungal activity (Figure 2). We thus conclude that the direct target of HT analogs is disruption of the fungal PM integrity and function.

\section{Lack of Resistance to HT Analog Antifungal Action}

Based on our findings that showed that HT analogs act directly and rapidly on the $\mathrm{PM}$, we presumed that resistance to this novel type of antifungals will be infrequent if any, similar to the case of other antimicrobials that target directly the periphery 

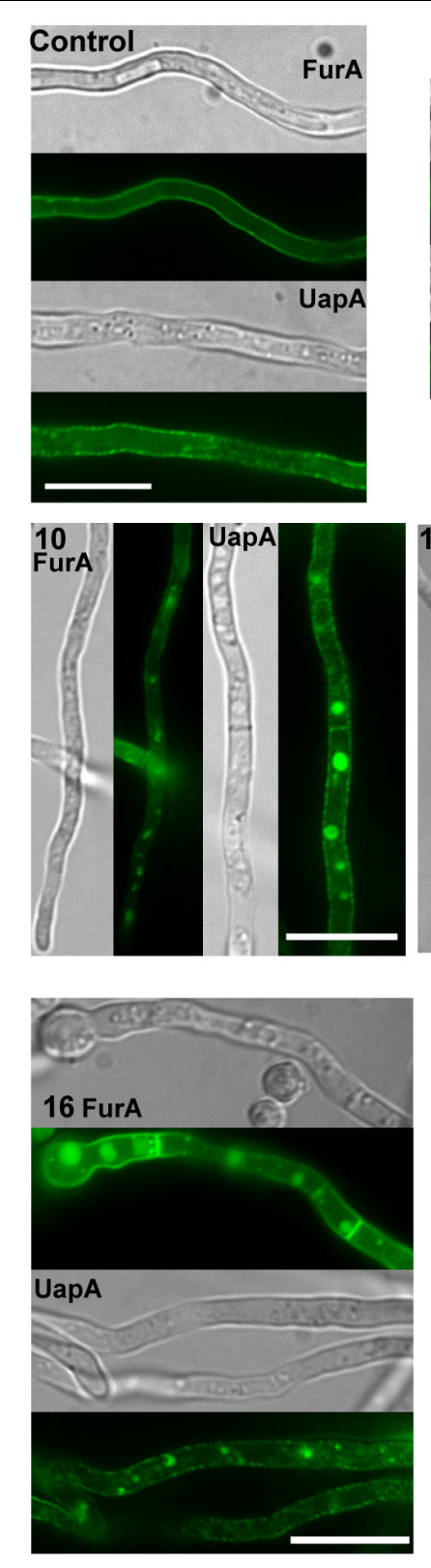
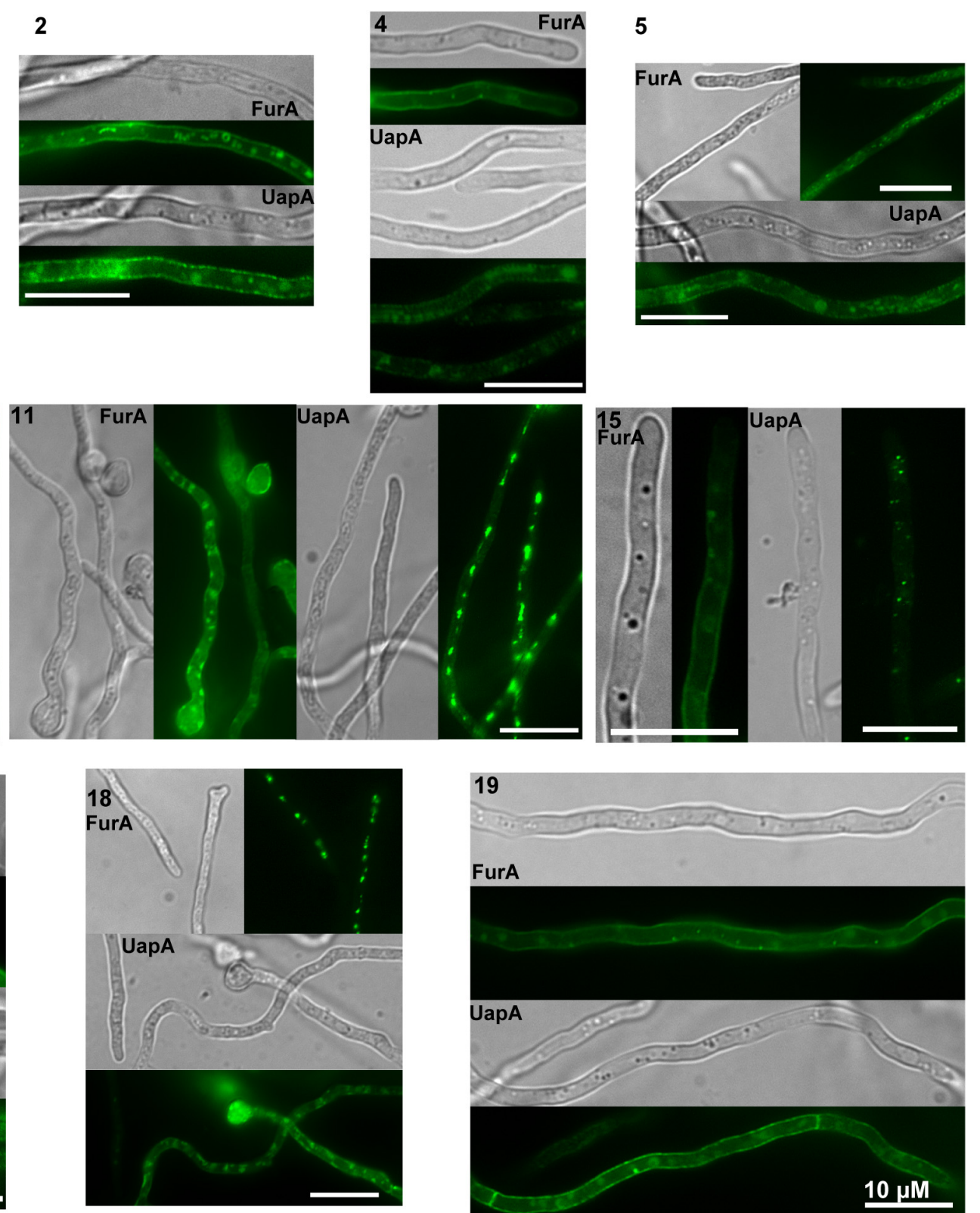

FIGURE 5 | Effect of HT analogs on A. nidulans plasma membrane and transporter-mediated uptake of xanthine. Epifluorescence in vivo microscopy showing the effect of HT analogs $(37.5 \mu \mathrm{M})$ on the plasma membrane of $A$. nidulans. The picture shows young hyphae of strains expressing functional GFP-tagged FurA or UapA transporters as PM molecular markers. FurA expression is stronger than that of UapA, due to transcription via a strong constitutive promoter ( $g p d A_{p}$ ), while UapA is transcribed by its native, relatively weak, promoter. Notice that upon addition of most analogs cortical fluorescent labeling is reduced with concurrent appearance of cytosolic fluorescent foci, which represent membrane aggregates. Scaleo shown are $10 \mu \mathrm{M}$.

of microbes, such as amphotericin B (plasma membrane) or Echinocandins (cell wall) (Cuenca-Estrella, 2014). To test this, we performed several experiments of standard u.v. or transposondriven mutagenesis as described in Materials and methods of an appropriate $A$. nidulans strain (i.e., one carrying the Minos transposable element (Evangelinos et al., 2015)), and tried to select mutant colonies resistant to $400 \mu \mathrm{M}$ of analog 15. We did not manage to isolate any resistance colony, even in cases were lethality of u.v. mutagenesis was $93.7 \%$. This apparently negative result is fully compatible with HT analogs targeting the PM of A. nidulans.

\section{DISCUSSION}

Here, we showed that specific chemically synthesized HT analogs possess broad and strong antifungal activity due to an immediate primary effect on the fungal plasma membrane. 


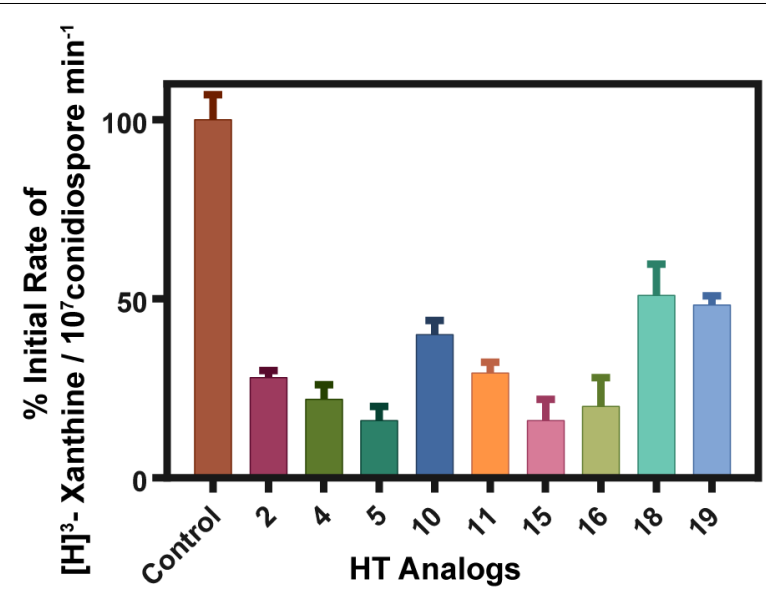

FIGURE 6 | Effect of HT analogs on transporter-mediated uptake of xanthine. $\%$ of initial uptake rates are shown as recorded in a standard $A$. nidulans strain. Analogs were added at final concentration of $100 \mu \mathrm{M} 10$ min prior to uptake measurements. Control stands for a sample where only the solvent DMSO was added at a concentration identical to the one used as a solvent in the analog samples. Control values were arbitrary taken as $100 \%$ rate. The results shown represent averages if three independent assays with $S D<15 \%$.

We do not, however, exclude that the HT analogs also have secondary cytotoxic targets (e.g., cell wall or enzymes), mostly in other microorganisms, which we did not test directly here. An additional finding classifies this specific set of compounds as very promising novel antifungals; lack of detectable fungal (A. nidulans) resistance due to mutations. The lack of obtaining spontaneous or mutation-induced resistance to an antifungal is a strong indication that the primary site of action of this antifungal is a molecule in the periphery of fungal cells, most probably a specific lipid of the PM. This is in full agreement with the effect detected herein of the HT analogs on the PM. Moreover, the lack of resistance resembles cases of other antifungals targeting the plasma membrane, such as amphotericin B, where only infrequent resistance is obtained due to mutations affecting the level of ergosterol and phospholipids in the membrane (CuencaEstrella, 2014; Fairlamb et al., 2016). Finally, an other aspect concerning the promising future of present HT analogs as novel antifungals is the observation that in preliminary tests analogs $\mathbf{1 5}$ and 16, which showed high antifungal activity, showed no toxicity against a mammalian cell line (see Materials and methods). Overall, our results open the way for the rigorous development of a novel, efficient and maybe safer class of antifungals. Additionally, the antifungal HT analogs were also shown to also possess antimicrobial activity against specific $\mathrm{G}+$ bacteria. This is an interesting, albeit rather preliminary, observation that we want to exploit in the future as it might give us information on the exact mechanism of action of interaction of these compounds with specific membrane lipids.

In several previous studies, HT has been shown to have variable antibacterial activity at relatively high concentrations, in the range of 1-4 $\mathrm{mM}$ (Capasso et al., 1995; Bisignano et al., 1999; Medina et al., 2006, 2007; Tafesh et al., 2011; Medina-Martínez et al., 2016). However, bacterial growth was never fully inhibited and a general conclusion on the effects of HT on Gram-positive or Gram-negative species could not be made (Medina-Martínez et al., 2016). To our knowledge, the only investigations regarding the antifungal potential of HT are those against medically important Candida spp., which showed minimal growth inhibitory values ranging between $633 \mu \mathrm{M}$ and $40 \mathrm{mM}$ (Pereira et al., 2007; Zoric et al., 2013). Notably, fluorescent dye-exclusion based studies with C. albicans revealed a membrane associated antifungal mechanism at subinhibitory concentrations (Zoric et al., 2013), in line with results presented herein. In the past, HT-derived compounds have been synthesized aiming at analogs with a better hydrophilic/lipophilic balance (HLB) to increase their cellular uptake and thus enhance their antioxidant or other unknown activities (Grasso et al., 2007; Bernini et al., 2012, 2015). However, no previous studies evaluating synthesized HT analogs as antifungal agents have been reported. HT derivatives have only recently been shown to have a very promising antitrypanosomal and antileishmanial activity (Belmonte-Reche et al., 2016). $\mathrm{IC}_{50}$ values against Trypanosoma brucei for HT decanoate ester and HT dodecanoate ester were 0.6 and $0.36 \mu \mathrm{M}$, respectively. This represented a significant 79132 fold improvement in activity compared to HT. Focusing on structure-activity relationships, the authors found via rational design several more HT analogs to have targeted cytotoxic activity against $T$. brucei with $\mathrm{IC}_{50}$ values in the low micromolar range. They concluded that the di-ortho phenolic ring and medium size alkyl chain are essential for activity, whereas the nature of the chemical bond among them seems less important. Importantly, antiprotozoan HT analogs displayed a high selectivity index against MRC-5, a non-tumoral human cell line, which is in line with the targeted antimicrobial specificity we also found for the HT antifungal analogs reported here. Thus, HT is indeed a highly promising mother compound to develop novel broad range antimicrobial agents.

Several of the new compounds described here showed varying degrees of antifungal activity against the tested pathogens, nevertheless compounds 2, 5, 11, 15, 16 and 19 possessed the strongest activity, clearly higher than the mother compound HT. All the active compounds belong to the first and second series, while compounds of the third series $(\mathbf{2 1}, \mathbf{2 2}, \mathbf{2 3}, \mathbf{2 4}$; see Supplementary Figure S1) were non-active. The carbonyl substituted compounds of the first series, and especially the alkyl-substituted analogs 2, 5 and 11, were among the most potent compounds, suggesting that the substitution on the $\alpha$-carbon is crucial for the activity. It seems that the presence of the hydroxyl group, is incompatible with activity, whereas the carbonyl group increases the antifungal capacity, probably by increasing the acidity of the catechol moiety. Notably also, all compounds in this study with an aromatic substitution (3, 6, 14 and 22; see Supplementary Figure S1) showed no activity against the tested pathogens, while the alkyl substituted compounds of first and second series were shown to be potent antifungals. The role of the double bond of the alkyl side chain is detrimental for the activity (see Supplementary Figure S1). Additionally, the role of the branching is not clear since the branched alkyl chained compounds 2, 5, 10, 11, 16, 18 and 19 showed potent antifungal activity, whereas compounds $\mathbf{9}$, 
12, 13 and 20 seem to be inactive. Nevertheless, our results demonstrate that only the alkyl substituted compounds of first and second series are active against the tested pathogens, with the most effective being those with an alkyl chain of 6 to 10 carbons. These results are also in agreement with the ones against Trypanosoma brucei for HT decanoate ester and HT dodecanoate ester (Belmonte-Reche et al., 2016).

According to recent data, alkyl gallates and other amphiphilic phenols, compounds possessing similar structure to the HT analogs presented here, have significant antifungal activity (Kozubek et al., 2001; Kubo et al., 2001b). It is suggested that their potency may result from the interaction with cell wall components (phospholipids, etc.); therefore, their activity is due partly to the ability of these molecules to function as nonionic surfactants (surface-active compounds), disrupting the fungi membrane. The mechanism by which surfactants exert their activity is not entirely understood, it is, however, hypothesized that, due to their amphiphilic character, they are inserted into the fungi lipid bilayer (possibly in other microbial membranes also), where they elicit an immediate destruction effect (Kubo et al., 2001a; Koziróg et al., 2018). In that aspect, the amphiphilic character of the HT analogs presented in this work and the experimental evidence for rapid destruction of the fungus membrane allow us to reasonably assume that they act primarily as nonionic surfactants, though other additional molecular interaction could account for the final effect. However, specific structural characteristics are needed for optimal antifungal activity strongly depended from a balance between the polar hydrophilic and the nonpolar hydrophobic part of the molecules. The aliphatic ester side chain (hydrophobic tail) is assumed to interfere with the cell membrane, while the acidity of the catechol group (hydrophilic head) seems to be essential for the antifungal activity. The initial contact with the lipid bilayer should involve the electrostatic interaction of the catechol system with the negatively charged phosphate groups of the membrane; thus the increased acidity of the hydroxyl groups due to the carbonyl substitution might be essential for activity. The divergence of the activity in relation to the structure of the alkyl tail indicates its vital role in the action of the compounds. The length (6 to 10 carbons) and increased volume because of alkyl substitutions are fundamental for optimum activity, whereas the substitution of the $\alpha$-carbon is critical for the electrostatic interaction of the hydrophilic head. The rationale for the role of the side alkyl chain is not entirely understood, and further research is needed in order to design more effective antifungal agents. Nevertheless, the fact that the HT analogs, very likely, targets the extra- cytoplasmic region could explain, as mentioned before, the lack of resistance of the described molecules.

Although recent technological advances made accessible unprecedented tools for drug discovery the development of new molecular scaffolds with demonstrated pharmacological properties is becoming slower and more expensive. Our study shows once more that the natural product chemical space provides the basis of successful design of new small-molecularweight molecules with excellent pharmacological properties. As
HT has been shown to undergo rapid oxidation the antifungal HT analogs described here will, however, need to be tested in vivo in mouse models and evaluated in respect to their stability or synergistic or antagonistic effects with other antifungals.

\section{MATERIALS AND METHODS}

\section{Synthesis of HT Analogs}

Chemical synthesis of HT analogs is described in detail in Supplementary Material. HT analogs were prepared in DMSO and aliquots and kept at $-20^{\circ} \mathrm{C}$. For experiments, the final concentration of DMSO in the medium was $<0.1 \%(\mathrm{v} / \mathrm{v})$ and the controls received DMSO only.

\section{Fungal and Bacterial Strains and Growth Media}

Standard "wild-type" fungal strains of A. nidulans (FGSC A4), A. fumigatus (Af293), A. flavus (NRRL3357), F. oxysporum (F3; (Christakopoulos et al., 1996)) and C. albicans clinical isolate from the Mycetotheca ATHUM collection of Athens University ${ }^{1}$ were used. The A. nidulans used contains a mutant allele (veA1) at the velvet locus, and a standard vitamin auxotrophy (pabA1 for para-aminobenzoic acid requirement). All other Aspergilli used correspond to standard strains used for genome sequencing ${ }^{2}$. For in vivo epifluorescence microscopy, strains expressing GFP-tagged functional UapA (Pantazopoulou et al., 2007) or FurA (Fernandez-Bolanos et al., 2012) transporters were used. Standard Aspergillus Minimal and Complete Media (MM and $\mathrm{CM}$ ) were used for growth of all fungi ${ }^{3}$. The nitrogen source used in $\mathrm{MM}$ was $10 \mathrm{mM} \mathrm{NaNO}_{3}$. Standard bacterial strains, coming from an in-house stock, of E. coli (DH5a), P. aeruginosa, P. fluorescens, Klebsiella sp., B. subtilis and S. aureus were used. Luria-Bertani medium was used for growth of bacterial strains.

\section{In vivo Evaluation of HT Analogs as Antifungals}

Fungal strains of A. nidulans, A. fumigatus, A. flavus, F. oxysporum and $C$. albicans were tested for their sensitivity/ resistance to different concentrations. Fungal spores of each strain were used to centrally inoculate a series of $35 \mathrm{~mm}$ petri dishes containing Minimal Media (MM) with $\mathrm{NaNO}_{3}$ as nitrogen source, in the presence or absence of various HT analog concentrations dissolved in DMSO. Controls without HT analogs contained solely DMSO. The final concentration range tested for toxicity of analogs in this work was $0-1000 \mu \mathrm{M}$. Figures shown highlight results using concentrations of 100 or $400 \mu \mathrm{M}$. Growth was followed after for 4-6 days, at 25 or $37^{\circ} \mathrm{C}$ and different $\mathrm{pH}$. Approximate concentrations leading to $50 \%$ reduction in the radial diameter of the growing colonies, $\left(I C_{50}\right)$, after 24 days (depending on the fungus) were recorded for each

\footnotetext{
${ }^{1}$ http://mycetotheca.biol.uoa.gr/

${ }^{2} \mathrm{http}$ //genome.jgi.doe.gov/programs/fungi/index.jsf

${ }^{3}$ http://www.fgsc.net
} 
analog. Approximate concentrations leading to no evident fungal growth were also recorded. For the non-filamentous fungus C. albicans we performed sensitivity tests in both liquid and solid cultures. For solid cultures testing, cells from a fresh liquid culture (O.D.600nm $=0.5$ at $10 \mathrm{~nm}$ ) were streaked on standard CM containing 100-400 $\mu \mathrm{M}$ of HT analogs, and incubated for 2 days at $37^{\circ} \mathrm{C}$. For liquid culture testing, HT analogs were added, at 100-400 $\mu \mathrm{M}$, at the start of the exponential phase (O.D.600nm $=0.5$ at $10 \mathrm{~nm}$ ) of a C. albicans culture and O.D.600nm measurements were recorded hourly for the next $6 \mathrm{~h}$, and after $24 \mathrm{~h}$. All final values shown in this article are averages of at least three independent experiments with no significant SD variation (e.g., $<20 \%)$.

\section{In vivo Evaluation of HT Analogs as Antibacterial Agents}

Standard bacterial strains of E. coli (DH5a), P. aeruginosa, P. fluorescens, Klebsiella sp., B. subtilis and S. aureus were tested for their sensitivity/resistance to different concentrations of compounds, which proved to act as antifungals. These tests were carried out either in solid or liquid LB media, in the presence or absence of $100-500 \mu \mathrm{M}$ of HT analogs. Tests on solid media were performed by recording single colony growth after bacterial streaking. Tests in liquid media were performed by recording O.D.600nm values $(10 \mathrm{~nm})$ after addition of $100-200 \mu \mathrm{M}$ of compounds in fresh exponentially growing bacterial cultures (O.D.600nm $=0.2-$ 0.4 ), and comparing these values to control cultures with no analogs. All final values shown in this article are averages of at least three independent experiments (SD variation $<15 \%$ ).

\section{In vivo Epifluorescence Microscopy}

Samples for standard inverted epifluorescence microscopy of A. nidulans strains were prepared as previously described (Martzoukou et al., 2017). In brief, germlings were incubated in sterile $35 \mathrm{~mm} \mu$-dishes, high glass bottom (ibidi, Germany) in $2 \mathrm{ml}$ liquid $\mathrm{MM}$ with $\mathrm{NaNO}_{3}$ as nitrogen source and the necessary vitamin supplements for $20 \mathrm{~h}$ at $25^{\circ} \mathrm{C}$. DMSO $(0.1 \%)$ or HT analogs (final concentrations tested 37.5 or $100 \mu \mathrm{M})$ dissolved in DMSO were added in samples under the microscope. Control samples where only DMSO was added (0.03-0.01\%) were also evaluated. Images were taken before and immediately after the addition of the analogs (or DMSO) and for a period of up to $30 \mathrm{~min}$. The strains used express the UapA or the FurA transporter as protein fluorescent markers specific for the plasma membrane (Pantazopoulou et al., 2007; Krypotou et al., 2015). Calcuofluor white staining of cell wall was as described in Martzoukou et al. (2017). Images were obtained with an AxioCa m HR R3 camera using the Zen lite 2012 software. Contrast adjustment, area selection and color combining were made using the Zen 2012 software. Images exported as tiffs were annotated and further processed in Adobe Photoshop CS4 Extended version 11.0.2 software for brightness adjustment, rotation and alignment.

\section{Transport Measurements}

Kinetic analysis- $\left[{ }^{3} \mathrm{H}\right]$-xanthine $(21.6 \mathrm{Ci} / \mathrm{mmol}$, Moravek Biochemicals, CA, United States) uptake in MM was assayed in germinating conidiospores of $A$. nidulans concentrated at $10^{7}$ conidiospores $/ 100 \mu \mathrm{L}$, at $37^{\circ} \mathrm{C}, \mathrm{pH} 6.8$, as previously described (Krypotou and Diallinas, 2014). Initial velocities were measured at $1 \mathrm{~min}$ of incubation with concentrations of $0.2-2.0 \mu \mathrm{M}$ of $\left[{ }^{3} \mathrm{H}\right]$-xanthine at the polarity maintenance stage $(3-4 \mathrm{~h}$, $130 \mathrm{rpm})$.

\section{Mutagenesis}

Two U.V. mutagenesis experiments, with lethality rates of 84.1 and $93.7 \%$, were performed at a standard distance of $20 \mathrm{~cm}$ from an Osram HNS30 UV-B/C lamp. $10^{9-10}$ conidiospores of a standard wild-type $A$. nidulans strain or a strain possessing a dual transposition system based on the Minos element (Evangelinos et al., 2015) were irradiated for $4 \mathrm{~min}$ and subsequently plated on MM plus nitrate medium containing $400 \mu \mathrm{M}$ of the HT analog 15. No colonies appeared after 1 -week incubation at $25^{\circ} \mathrm{C}$.

\section{Toxicity in a Mammalian Cell Line}

N2A mouse neuroblastoma cells were used, in a standard MTT assay (Berridge et al., 2005), to test the whether antifungal HT analogs elicit cytotoxicity in a standard mammalian cell line. Analogs 15 or 16 were tested at two concentrations (100 or $400 \mu \mathrm{M}$ ) as described in materials and methods. The N2A mouse neuroblastoma cells were grown in Dulbecco's Modified Eagle's Medium (DMEM) that contained 10\% fetal bovine serum, 1\% of penicillin and streptomycin in 96-well plates at a density of 15,000 cells/well. A standard MTT assay was used to assess cell metabolic activity in the absence (addition of solely DMSO) and presence of HT analogs (Berridge et al., 2005). The cultures were grown for 6 days at $37^{\circ} \mathrm{C}$ with $5 \% \mathrm{CO}_{2}$. Then the medium was changed to one containing 100 or $400 \mu \mathrm{M}$ of 15 or 16 and incubated for $24 \mathrm{~h}$ at $37^{\circ} \mathrm{C}$ with $5 \% \mathrm{CO}_{2}$. In all cases the final concentration of DMSO was $\leq 0.1 \%$. $20 \mu \mathrm{l}$ of the dye MTT $(2.5 \mathrm{mg} / \mathrm{ml}$ MTT in PBS) was added to each well and incubated for $4 \mathrm{~h}$. The resulting formazan dye was extracted with $100 \mu \mathrm{l}$ isopropanol/ $\mathrm{HCl}(100 \mathrm{ml}$ isopropanol $+833 \mu \mathrm{l}$ $\mathrm{HCl}$ ) and the absorbance was measured spectrophotometrically at a wavelength of $545 \mathrm{~nm}$. Statistical analysis: All experiments were repeated three times. One-way ANOVA with Bonferroni's Multiple Comparison Test was used to evaluate the statistical significance of the differences. Statistical significance was defined as $p<0.05$.

\section{AUTHOR CONTRIBUTIONS}

GD performed and designed the biological experiments, analyzed the results and wrote the manuscript. NR performed the biological experiments and made relevant figures. VT and IZ performed the biological experiments. IK and AK performed the chemical experiments. EM and AS analyzed the results. IKK designed the chemical synthesis, analyzed the results and wrote the manuscript. 


\section{FUNDING}

This work was partly supported by a "Stavros Niarchos Foundation" research grant and by "Fondation Santé."

\section{ACKNOWLEDGMENTS}

This work has been released as a pre-print at BioRxiv (Diallinas et al., 2018). We thank Dr. Joseph Meletiadis, Assistant Professor

\section{REFERENCES}

Arendrup, M. C. (2014). Update on antifungal resistance in Aspergillus and Candida. Clin. Microbiol. Infect. 20(Suppl. 6), 42-48. doi: 10.1111/1469-0691. 12513

Belmonte-Reche, E., Martínez-García, M., Peñalver, P., Gómez-Pérez, V., Lucas, R., Gamarro, F., et al. (2016). Tyrosol and hydroxytyrosol derivatives as antitrypanosomal and antileishmanial agents. Eur. J. Med. Chem. 119, 132-140. doi: 10.1016/j.ejmech.2016.04.047

Bernini, R., Crisante, F., Barontini, M., Tofani, D., Balducci, V., and Gambacorta, A. (2012). Synthesis and structure/antioxidant activity relationship of novel catecholic antioxidant structural analogues to hydroxytyrosol and its lipophilic esters. J. Agric. Food Chem. 60, 7408-7416. doi: $10.1021 /$ jf301131a

Bernini, R., Gilardini Montani, M. S., Merendino, N., Romani, A., and Velotti, F. (2015). Hydroxytyrosol-derived compounds: a basis for the creation of new pharmacological agents for cancer prevention and therapy. J. Med. Chem. 58, 9089-9107. doi: 10.1021/acs.jmedchem.5b00669

Berridge, M. V., Herst, P. M., and Tan, A. S. (2005). Tetrazolium dyes as tools in cell biology: new insights into their cellular reduction. Biotechnol. Annu. Rev. 11, 127-152. doi: 10.1016/S1387-2656(05)11004-7

Bisignano, G., Tomaino, A., Lo Cascio, R., Crisafi, G., Uccella, N., and Saija, A. (1999). On the in-vitro antimicrobial activity of oleuropein and hydroxytyrosol. J. Pharm. Pharmacol. 51, 971-974. doi: 10.1211/0022 357991773258

Capasso, R., Evidente, A., Schivo, L., Orru, G., Marcialis, M. A., and Cristinzio, G. (1995). Antibacterial polyphenols from olive oil mill waste waters. J. Appl. Bacteriol. 79, 393-398. doi: 10.1111/j.1365-2672.1995.tb03153.x

Chillemi, R., Sciuto, S., Spatafora, C., and Tringali, C. (2010). "Hydroxytyrosol lipophilic analogues: synthesis, radical scavenging activity and human cell oxidative damage protection," in Olives and Olive Oil in Health and Disease Prevention, eds V. Preedy and R. Watson (New York, NY: Academic Press), 1233-1243. doi: 10.1016/B978-0-12-374420-3.00135-2

Christakopoulos, P., Nerinckx, W., Kekos, D., Macris, B., and Claeyssens, M. (1996). Purification and characterization of two low molecular mass alkaline xylanases from Fusarium oxysporum F3. J. Biotechnol. 51, 181-189. doi: 10. 1016/0168-1656(96)01619-7

Cuenca-Estrella, M. (2014). Antifungal drug resistance mechanisms in pathogenic fungi: from bench to bedside. Clin. Microbiol. Infect. 20(Suppl. 6), 54-59. doi: 10.1111/1469-0691.12495

Di Santo, R. (2010). Natural products as antifungal agents against clinically relevant pathogens. Nat. Prod. Rep. 27, 1084-1098. doi: 10.1039/b914961a

Diallinas, G., Rafailidou, N., Kalpaktsi, I., Komianou, A. C., Tsouvali, V., Zantza, I., et al. (2018). Hydroxytyrosol (HT) analogues act as potent antifungals by direct disruption of the fungal cell membrane. bioRxiv [Preprint]. doi: 10.1101/350025

Evangelinos, M., Anagnostopoulos, G., Karvela-Kalogeraki, I., Stathopoulou, P. M., Scazzocchio, C., and Diallinas, G. (2015). Minos as a novel Tc1/mariner-type transposable element for functional genomic analysis in Aspergillus nidulans. Fungal Genet. Biol. 81, 1-11. doi: 10.1016/j.fgb.2015.05.007

Fairlamb, A. H., Gow, N. A., Matthews, K. R., and Waters, A. P. (2016). Drug resistance in eukaryotic microorganisms. Nat. Microbiol. 1:16092. doi: 10.1038/ nmicrobiol.2016.92

Fernandez-Bolanos, J. G., Lopez, O., Fernandez-Bolanos, J., and RodriguezGutierrez, G. (2008). Hydroxytyrosol and derivatives: isolation, synthesis of Microbiology in the Medical School of University of Athens, for Amphotericin B and Michalis Papadourakis for help in preliminary uptake experiments.

\section{SUPPLEMENTARY MATERIAL}

The Supplementary Material for this article can be found online at: https://www.frontiersin.org/articles/10.3389/fmicb. 2018.02624/full\#supplementary-material

and biological properties. Curr. Org. Chem. 12, 442-463. doi: 10.2174/ 138527208784083888

Fernandez-Bolanos, J. G., Lopez, O., Lopez-Garcia, M. A., and Marset, A. (2012). "Biological properties of hydroxytyrosol and its derivatives," in Olive OilConstituents, Quality, Health Properties and Bioconversions, ed. D. Boskou (Rijeka: InTech Europe), 375-396.

Grasso, S., Siracusa, L., Spatafora, C., Renis, M., and Tringali, C. (2007). Hydroxytyrosol lipophilic analogues: enzymatic synthesis, radical scavenging activity and DNA oxidative damage protection. Bioorg. Chem. 35, 137-152. doi: 10.1016/j.bioorg.2006.09.003

Köhler, J. R., Hube, B., Puccia, R., Casadevall, A., and Perfect, J. R. (2017). Fungi that infect humans. Microbiol. Spectr. 5:FUNK-0014-2016. doi: 10.1128/ microbiolspec.FUNK-0014-2016

Koolen, H. H., Pral, E. M., Alfieri, S. C., Marinho, J. V., Serain, A. F., Hernández-Tasco, A. J., et al. (2017). Antiprotozoal and antioxidant alkaloids from Alternanthera littoralis. Phytochemistry 134, 106-113. doi: 10.1016/j. phytochem.2016.11.008

Korukluoglu, M., Sahan, Y., and Yigit, A. (2008). Antifungal properties of olive leaf extracts and their phenolic compounds. J. Food Saf. 28, 76-87. doi: 10.1111/j. 1745-4565.2007.00096.x

Koziróg, A., Brycki, B., and Pielech-Przybylska, K. (2018). Impact of cationic and neutral gemini surfactants on conidia and hyphal forms of Aspergillus brasiliensis. Int. J. Mol. Sci. 19:E873. doi: 10.3390/ijms 19030873

Kozubek, A., Zarnowski, R., Stasiuk, M., and Gubernator, J. (2001). Natural amphiphilic phenols as bioactive compounds. Cell. Mol. Biol. Lett. 6, 351-355.

Krypotou, E., and Diallinas, G. (2014). Transport assays in filamentous fungi: kinetic characterization of the UapC purine transporter of Aspergillus nidulans. Fungal Genet. Biol. 63, 1-8. doi: 10.1016/j.fgb.2013.12.004

Krypotou, E., Evangelidis, T., Bobonis, J., Pittis, A. A., Gabaldón, T., Scazzocchio, C., et al. (2015). Origin, diversification and substrate specificity in the family of NCS1/FUR transporters. Mol. Microbiol. 96, 927-950. doi: $10.1111 / \mathrm{mmi} .12982$

Kubo, I., Fujita, K., and Lee, S. H. (2001a). Antifungal mechanism of polygodial. J. Agric. Food Chem. 49, 1607-1611. doi: 10.1021/jf000136g

Kubo, I., Xiao, P., and Fujita, K. (2001b). Antifungal activity of octyl gallate: structural criteria and mode of action. Bioorg. Med. Chem. Lett. 11, 347-350. doi: 10.1016/S0960-894X(00)00656-9

Li, F. S., and Weng, J. K. (2017). Demystifying traditional herbal medicine with modern approach. Nat. Plants 3:17109. doi: 10.1038/nplants.2017.109

Low, C. Y., and Rotstein, C. (2011). Emerging fungal infections in immunocompromised patients. F1000 Med. Rep. 3:14. doi: 10.3410/M3-14

Manna, C., Migliardi, V., Sannino, F., De Martino, A., and Capasso, R. (2005). Protective effects of synthetic hydroxytyrosol acetyl derivatives against oxidative stress in human cells. J. Agric. Food Chem. 53, 9602-9607. doi: 10. 1021/jf058110i

Martzoukou, O., Amillis, S., Zervakou, A., Christoforidis, S., and Diallinas, G. (2017). The AP-2 complex has a specialized clathrin-independent role in apical endocytosis and polar growth in fungi. eLife 6:e20083. doi: 10.7554/eLife. 20083

McCarthy, M. W., Kontoyiannis, D. P., Cornely, O. A., Perfect, J. R., and Walsh, T. J. (2017). Novel agents and drug targets to meet the challenges of resistant fungi. J. Infect. Dis. 216(Suppl._3), S474-S483. doi: 10.1093/infdis/ jix130 
Medina, E., Brenes, M., Romero, C., García, A., and de Castro, A. (2007). Main antimicrobial compounds in table olives. J. Agric. Food Chem. 55, 9817-9823. doi: 10.1021/jf0719757

Medina, E., de Castro, A., Romero, C., and Brenes, M. (2006). Comparison of the concentrations of phenolic compounds in olive oils and other plant oils: correlation with antimicrobial activity. J. Agric. Food Chem. 54, 4954-4961. doi: $10.1021 /$ jf0602267

Medina-Martínez, M. S., Truchado, P., Castro-Ibáñez, I., and Allende, A. (2016). Antimicrobial activity of hydroxytyrosol: a current controversy. Biosci. Biotechnol. Biochem. 80, 801-810. doi: 10.1080/09168451.2015.1116924

Odds, F. C., Brown, A. J., and Gow, N. A. (2003). Antifungal agents: mechanisms of action. Trends Microbiol. 11, 272-279. doi: 10.1016/S0966-842X(03) 00117-3

Pan, S. Y., Zhou, S. F., Gao, S. H., Yu, Z. L., Zhang, S. F., Tang, M. K., et al. (2013). New perspectives on how to discover drugs from herbal medicines: CAM's outstanding contribution to modern therapeutics. Evid. Based Complement. Altern. Med. 2013:627375. doi: 10.1155/2013/627375

Pantazopoulou, A., and Diallinas, G. (2007). Fungal nucleobase transporters. FEMS Microbiol. Rev. 31, 657-675. doi: 10.1111/j.1574-6976.2007.00083.x

Pantazopoulou, A., Lemuh, N. D., Hatzinikolaou, D. G., Drevet, C., Cecchetto, G., Scazzocchio, C., et al. (2007). Differential physiological and developmental expression of the UapA and AzgA purine transporters in Aspergillus nidulans. Fungal Genet. Biol. 44, 627-640. doi: 10.1016/j.fgb.2006.10.003

Pereira, A. P., Ferreira, I. C., Marcelino, F., Valentão, P., Andrade, P. B., Seabra, R., et al. (2007). Phenolic compounds and antimicrobial activity of olive (Olea europaea L. Cv. Cobrançosa) leaves. Molecules 12, 1153-1162. doi: 10.3390/ 12051153

Robles-Almazan, M., Pulido-Moran, M., Moreno-Fernandez, J., RamirezTortosa, C., Rodriguez-Garcia, C., Quiles, J. L., et al. (2018). Hydroxytyrosol: bioavailability, toxicity, and clinical applications. Food Res. Int. 105, 654-667. doi: 10.1016/j.foodres.2017.11.053

Tafesh, A., Najami, N., Jadoun, J., Halahlih, F., Riepl, H., and Azaizeh, H. (2011). Synergistic antibacterial effects of polyphenolic compounds from olive mill wastewater. Evid. Based Complement. Altern. Med. 2011:431021. doi: 10.1155/ 2011/431021

Tasleem, A., Bhosale, J. D., Naresh, K., Mandal, T. K., Bendre, R. S., Lavekar, G. S., et al. (2009). Natural products-antifungal agents derived from plants. J. Asian Nat. Prod. Res. 11, 621-638. doi: 10.1080/10286020902942350

Teodoro, G., Ellepola, K., Senevirante, C. J., and Koga-Ito, C. Y. (2015). Potential use of phenolic acids as anti-candida agents: a review. Front. Microbiol. 6:1420. doi: $10.3389 /$ fmicb.2015.01420

Thielmann, J., Kohnen, S., and Hauser, C. (2017). Antimicrobial activity of Olea europaea Linné extracts and their applicability as natural food preservative agents. Int. J. Food Microbiol. 251, 48-66. doi: 10.1016/j.ijfoodmicro.2017.03.019

Walter, W. M. Jr., Flemming, H. P., and Etchells, J. L. (1973). Preparation of antimicrobial compounds by hydrolysis of oleuropein fromgreen olives. Appl. Microbiol. 26, 773-776.

Zoric, N., Horvat, I., Kopjar, N., Vucemilovic, A., Kremer, D., Tomic, S., et al. (2013). Hydroxytyrosol expresses antifungal activity in vitro. Curr. Drug Targets 14, 992-998. doi: 10.2174/13894501113149990167

Zorić, N., Kopjar, N., Bobnjarić, I., Horvat, I., Tomić, S., and Kosalec, I. (2016). Antifungal activity of oleuropein against Candida albicans-the in vitro study. Molecules 21:1631. doi: 10.3390/molecules21121631

Conflict of Interest Statement: The authors declare that the research was conducted in the absence of any commercial or financial relationships that could be construed as a potential conflict of interest.

Copyright (c) 2018 Diallinas, Rafailidou, Kalpaktsi, Komianou, Tsouvali, Zantza, Mikros, Skaltsounis and Kostakis. This is an open-access article distributed under the terms of the Creative Commons Attribution License (CC BY). The use, distribution or reproduction in other forums is permitted, provided the original author(s) and the copyright owner(s) are credited and that the original publication in this journal is cited, in accordance with accepted academic practice. No use, distribution or reproduction is permitted which does not comply with these terms. 